\title{
Pretorsion theories, stable category and preordered sets
}

\author{
Alberto Facchini $^{1}$ (D) Carmelo Antonio Finocchiaro ${ }^{1,2}$
}

Received: 22 July 2019 / Accepted: 13 September 2019 / Published online: 25 September 2019 (c) Fondazione Annali di Matematica Pura ed Applicata and Springer-Verlag GmbH Germany, part of Springer Nature 2019

\begin{abstract}
We show that in the category of preordered sets there is a natural notion of pretorsion theory, in which the partially ordered sets are the torsion-free objects and the sets endowed with an equivalence relation are the torsion objects. Correspondingly, it is possible to construct a stable category factoring out the objects that are both torsion and torsion-free.
\end{abstract}

Keywords Preorder · Torsion theory $\cdot$ Stable category

Mathematics Subject Classification Primary 06A06 - 06A11; Secondary 08A99 · 18B99

\section{Introduction}

Every preorder can be obtained from an equivalence relation and a partial order. In this paper, we show that this can be interpreted in the language of a suitably defined (pre)torsion theory. More precisely, we consider the category Preord of all preordered sets $(A, \rho)$ and its full subcategories ParOrd and Equiv, whose objects are all partially ordered sets $(A, \leq)$ and all sets $(A, \sim)$, where $\sim$ is an equivalence relation on $A$, respectively. Call trivial objects of Preord the objects of Preord that are in both ParOrd and Equiv. Trivial objects are the objects of Preord of the form $(A,=)$, where $=$ is the equality relation on $A$. Call trivial morphisms the morphisms in Preord that factor through a trivial object. Factoring out the

The first author was partially supported by Dipartimento di Matematica "Tullio Levi-Civita" of Università di Padova (Project BIRD163492/16 "Categorical homological methods in the study of algebraic structures" and Research Program DOR1828909 "Anelli e categorie di moduli”). The second author was partially supported by GNSAGA of Istituto Nazionale di Alta Matematica, by Dipartimento di Matematica "Tullio Levi-Civita" of Università di Padova (Research Program DOR1828909 “Anelli e categorie di moduli”), and by Dipartimento di Matematica e Informatica, Università di Catania (Research Program "Proprietà algebriche locali e globali di anelli associati a curve e ipersuperfici” PTR 2016-18).

Alberto Facchini

facchini@math.unipd.it

Carmelo Antonio Finocchiaro

cafinocchiaro@unict.it

1 Dipartimento di Matematica "Tullio Levi-Civita", Università di Padova, 35121 Padova, Italy

2 Dipartimento di Matematica e Informatica, Università di Catania, 95125 Catania, Italy 
category Preord modulo the trivial objects and the trivial morphisms, we get a category Preord, called the stable category of Preord. The category Preord minus the empty object, denoted by Preord*, turns out to be a pointed category, that is, a category with a zero object. In the pointed category Preord ${ }^{*}$, we have the notions of kernel and cokernel, which have their natural counterpart in Preord. We call these natural counterparts prekernels and precokernels. This allows us to define short preexact sequences: if $f: A \rightarrow B$ and $g: B \rightarrow C$ are two morphisms in Preord, we say that $A \stackrel{f}{\longrightarrow} B \stackrel{g}{\longrightarrow} C$ is a short preexact sequence in Preord if $f$ is a prekernel of $g$ and $g$ is a precokernel of $f$. For example, for every object $(A, \rho)$ in Preord, let $\sim$ be an equivalence relation on $A$ contained in $\rho$. Then

$$
(A, \sim) \stackrel{k}{\longrightarrow}(A, \rho) \stackrel{\pi}{\longrightarrow}(A / \sim, \rho)
$$

is a short preexact sequence in Preord. Here $k$ is the identity mapping and $\pi$ is the canonical projection. For any partially ordered set $(A, \rho)$, let $\simeq{ }_{\rho}$ denote the equivalence relation on $A$ defined, for every $a, b \in A$, by $a \simeq{ }_{\rho} b$ if $a \rho b$ and $b \rho a$. Then

$$
(A, \simeq \rho) \stackrel{k}{\longrightarrow}(A, \rho) \stackrel{\pi}{\longrightarrow}\left(A / \simeq_{\rho}, \leq_{\rho}\right)
$$

is a short preexact sequence in Preord, $\left(A, \simeq_{\rho}\right) \in$ Equiv and $\left(A / \simeq_{\rho}, \leq_{\rho}\right) \in$ ParOrd. It is now easy to generalize what we have seen above and define pretorsion theories for arbitrary categories $\mathcal{C}$. Our definition is as follows. Let $\mathcal{C}$ be an arbitrary category. A pretorsion theory $(\mathcal{T}, \mathcal{F})$ for $\mathcal{C}$ consists of two classes $\mathcal{T}, \mathcal{F}$ of objects of $\mathcal{C}$, closed under isomorphism, satisfying the following two conditions. Set $\mathcal{Z}:=\mathcal{T} \cap \mathcal{F}$, let $\operatorname{Triv}_{\mathcal{Z}}(X, Y)$ be the set of all morphisms $X \rightarrow Y$ in $\mathcal{C}$ that factor though an object of $\mathcal{Z}$, and call $\mathcal{Z}$-preexact any sequence preexact relatively to $\mathcal{Z}$. The two conditions sufficient to define our pretorsion theories $(\mathcal{T}, \mathcal{F})$ are:

(1) For every object $B$ of $\mathcal{C}$ there is a short $\mathcal{Z}$-preexact sequence

$$
A \stackrel{f}{\longrightarrow} B \stackrel{g}{\longrightarrow} C
$$

with $A \in \mathcal{T}$ and $C \in \mathcal{F}$.

(2) $\operatorname{Hom}_{\mathcal{C}}(T, F)=\operatorname{Triv}_{\mathcal{Z}}(T, F)$ for every object $T \in \mathcal{T}, F \in \mathcal{F}$.

The pair (Equiv, PreOrd) turns out to be a pretorsion theory in Preord.

The category Preord* provides an example of a category that arises as a quotient category of a topological category (the category of Alexandroff-discrete spaces, i.e. of preordered sets), but nevertheless has features typical of categories of commutative algebra (short exact sequences).

\section{Preorders, partial orders and equivalence relations}

A preorder on a set $A$ is a relation on $A$ that is reflexive and transitive. We will denote by Preord the category of all preordered sets. Its objects are the pairs $(A, \rho)$, where $A$ is a set and $\rho$ is a preorder on $A$. The morphisms $f:(A, \rho) \rightarrow\left(A^{\prime}, \rho^{\prime}\right)$ in Preord are the mappings $f$ of $A$ into $A^{\prime}$ such that $a \rho b$ implies $f(a) \rho^{\prime} f(b)$ for all $a, b \in A$. As usual, when there is no danger of confusion or when the preorder is clear from the context, we will denote the preordered set $(A, \rho)$ simply by $A$.

Remark 2.1 We now fix some trivial notations useful in the sequel. The category Preord has arbitrary coproducts and arbitrary products. Given any family $\left\{\left(A_{i}, \rho_{i}\right) \mid i \in I\right\}$ of 
preordered sets, the coproduct of the family is the disjoint union $A=\bigsqcup_{i \in I} A_{i}(:=\{(a, i) \mid$ $\left.\left.i \in I, a \in A_{i}\right\}\right)$, endowed with the coproduct preorder $\rho$ defined, for every $(a, i),(b, j) \in A$, by $(a, i) \rho(b, j)$ if $i=j$ and $a \rho_{i} b$. The product in Preord of the family is its Cartesian product with the componentwise preorder.

The main examples of preordered sets $(A, \rho)$ are those in which the preorder $\rho$ is a partial order (i.e. $\rho$ is antisymmetric) or an equivalence relation (i.e. $\rho$ is symmetric). The full subcategories of Preord whose objects are all preordered sets $(A, \rho)$ with $\rho$ a partial order (an equivalence relation) will be denoted by ParOrd (Equiv, respectively).

In our first proposition, we describe preorders on a set $A$. They are obtained from an equivalence relation $\sim$ on $A$ and a partial order on the quotient set $A / \sim$.

Proposition 2.2 Let A be a set. There is a one-to-one correspondence between the set of all preorders $\rho$ on $A$ and the set of all pairs $(\sim, \leq)$, where $\sim$ is an equivalence relation on $A$ and $\leq$ is a partial order on the quotient set $A / \sim$. The correspondence associates with every preorder $\rho$ on A the pair $\left(\simeq_{\rho}, \leq_{\rho}\right)$, where $\simeq_{\rho}$ is the equivalence relation defined, for every $a, b \in A$, by $a \simeq \simeq_{\rho} b$ if $a \rho b$ and $b \rho a$, and $\leq_{\rho}$ is the partial order on $A / \simeq_{\rho}$ defined, for every $a, b \in A$, by $[a]_{\simeq_{\rho}} \leq[b]_{\simeq_{\rho}}$ if a $\rho b$. Conversely, for any pair $(\sim, \leq)$ with $\sim$ an equivalence relation on $A$ and $\leq$ a partial order on $A / \sim$, the corresponding preorder $\rho_{(\sim, \leq)}$ on $A$ is defined, for every $a, b \in A$, by $a \rho_{(\sim, \leq)} b$ if $[a]_{\sim} \leq[b]_{\sim}$.

The objects of Preord that are objects in both the full subcategories ParOrd and Equiv are the objects of the form $(A,=)$, where $=$ denotes the equality relation on $A$. We will call them the trivial objects of Preord. The full subcategory of Preord whose objects are all trivial objects $(A,=)$ is isomorphic to the category of sets.

A morphism $f:(A, \rho) \rightarrow\left(A^{\prime}, \rho^{\prime}\right)$ in Preord is trivial if it factors through a trivial object, that is, if there exist a trivial object $(B,=)$ and morphisms $g:(A, \rho) \rightarrow(B,=)$ and $h:(B,=) \rightarrow\left(A^{\prime}, \rho^{\prime}\right)$ in Preord with $f=h g$. The following lemma has an easy proof:

Lemma 2.3 Let $(A, \rho)$ and $\left(A^{\prime}, \rho^{\prime}\right)$ be objects in the category Preord and $f: A \rightarrow A^{\prime}$ be a morphism. Then $f:(A, \rho) \rightarrow\left(A^{\prime}, \rho^{\prime}\right)$ is a trivial morphism in Preord if and only if a $\rho b$ implies $f(a)=f(b)$ for all $a, b \in A$.

We will denote by $\operatorname{Hom}\left(A, A^{\prime}\right)$ the set of all morphisms $A \rightarrow A^{\prime}$ in the category Preord and by $\operatorname{Triv}\left(A, A^{\prime}\right)$ the set of all trivial morphisms $A \rightarrow A^{\prime}$.

In order to justify the terminology that follows, it is now convenient to introduce some notions of topology. Recall that a topological space is Alexandroff-discrete provided that the intersection of any family of open subsets is an open subset. The full subcategory of Top whose objects are all Alexandroff-discrete spaces is isomorphic to the category Preord. The category isomorphism associates with any preordered set $(X, \rho)$, the topological space $\left(X, \tau_{\rho}\right)$, whose open sets are the subsets $A$ of $X$ such that, if $a \in A, x \in X$ and $x \rho a$, then $x \in A$. It is immediately seen that $\left(X, \tau_{\rho}\right)$ is an Alexandroff-discrete space. Moreover, for any $x, y \in X, x \rho y$ if and only if $y$ belongs to the closure $\overline{\{x\}}$ of $x$ with respect to the topology $\tau_{\rho}$. A mapping $X \rightarrow Y$ is a morphism $(X, \rho) \rightarrow(Y, \sigma)$ in Preord if and only if it is a continuous mapping $\left(X, \tau_{\rho}\right) \rightarrow\left(Y, \tau_{\sigma}\right)$. Conversely, let $(X, \tau)$ be any Alexandroff-discrete space. The associated preorder $\leq_{\tau}$ on $X$ is defined, for every, $x, y \in X$, by $x \leq_{\tau} y$ if $\overline{\{y\}} \subseteq \overline{\{x\}}$.

It is easy to see that:

Proposition 2.4 A morphism $f:(A, \rho) \rightarrow\left(A^{\prime}, \rho^{\prime}\right)$ in Preord is a monomorphism in Preord if and only if it is an injective mapping, and is an epimorphism if and only if it is a surjective mapping. 
Remark 2.5 Trivial objects are exactly the projective objects in Preord. Also, all trivial objects $(X,=)$ with $X \neq \varnothing$ are (projective) generators in Preord. These two facts can be easily checked directly. Also see [1].

Now we define a suitable quotient category of the category Preord, which we will call the stable category and denote by Preord. As far as quotient categories are concerned, we follow [9, pp. 51-52]. For every object $(A, \rho)$ of Preord, we will say that a subset $B$ of $A$ is a clopen subset if, for every $a \in A \backslash B$ and $b \in B, a \not \varnothing b$ and $b \not \varnothing a$.

Remarks 2.6 (1) Let $(A, \rho)$ be a preordered set, and let $B \subseteq A$. By definition, $B$ is a clopen subset, according to the definition just given, if and only if $B$ is a clopen subset in the Alexandroff-discrete space $\left(A, \tau_{\rho}\right)$.

(2) Let $\left(A_{i}, \rho_{i}\right), i \in I$, be preordered sets, and let $\left(\coprod_{i \in I} A_{i}, \rho\right)$ be their coproduct in Preord. Fix an index $i \in I$. By definition, if $j \in I \backslash\{i\}, a \in A_{i}$ and $b \in A_{j}$, then $(a, i) \not \supset(b, j)$ and $(b, j) \not \supset(a, i)$. Hence the canonical image $A_{i} \times\{i\}$ of $A_{i}$ in the coproduct is a clopen subset of $\bigsqcup_{i \in I} A_{i}$.

Definition 2.7 Let $A$ be a preordered set. We say that $A$ is indecomposable in Preord if $A$ is non-empty, and for any preordered sets $B, C$, we have that $A \cong B \coprod C$ in Preord implies $B=\emptyset$ or $C=\emptyset$. (An equivalent definition of indecomposable, or connected, object which makes sense in any category $\mathcal{C}$ is the following: an object $A$ is indecomposable: whenever $A \cong \bigsqcup_{i \in I} B_{i}$, then there is an index $i \in I$ for which the coproduct injection $B_{i} \rightarrow A$ is an isomorphism. Notice that the case $I=\emptyset$ shows that such $A$ cannot be initial in the category C.)

From Remark 2.6(2), we get that:

Lemma 2.8 The following conditions are equivalent for a non-empty preordered $\operatorname{set}(A, \rho)$ :

1. $(A, \rho)$ is indecomposable.

2. The Alexandroff-discrete space $\left(A, \tau_{\rho}\right)$ is connected, that is, its clopen subsets are only $\emptyset$ and $A$.

For any preordered set $(A, \rho)$, let $\equiv \rho$ be the equivalence relation generated by $\rho$, that is, the transitive closure of the relation $\rho \rho^{o}$ (i.e. $x \equiv \rho y$ if and only if there exist elements $x_{0}=x, x_{1}, \ldots, x_{n}=y \in A$ such that, for every $i=1,2, \ldots n, x_{i-1} \rho x_{i}$ or $\left.x_{i} \rho x_{i-1}\right)$.

The proof of the following lemma is elementary:

Lemma 2.9 Let $(A, \rho)$ be a preordered set.

1. If $B \subseteq A$ is clopen and $a \in B$, then $[a]_{\equiv_{\rho}} \subseteq B$.

2. For any $a \in A$, the equivalence class $[a]_{\equiv_{\rho}}$ is clopen and connected.

3. Every clopen subset of $A$ is a union of equivalence classes modulo $\equiv_{\rho}$.

4. The connected components of $A$ are exactly the equivalence classes of A modulo $\equiv_{\rho}$.

Proof (1) Suppose $x \in[a]_{\equiv_{\rho}}$. By definition, there are elements $x_{0}, x_{1}, \ldots, x_{n} \in A$ such that $a=x_{0}, x=x_{n}$, and for every $i=1,2,3, \ldots, n, x_{i-1} \rho x_{i}$ or $x_{i} \rho x_{i-1}$. Since $a \in B$ and either $a \rho x_{1}$ or $x_{1} \rho a$, the fact that $B$ is clopen implies that $x_{1} \in B$. Then $x \in B$ by induction.

(2) Assume $x \in[a]_{\equiv_{\rho}}$ and $y \in A$. If $x \rho y$ or $y \rho x$, then $x \equiv_{\rho} y$, since $\equiv_{\rho}$ is generated by $\rho$. It follows $y \in[a]_{\equiv_{\rho}}$, and this proves that $[a]_{\equiv_{\rho}}$ is clopen. Suppose there are non-empty clopen sets $U, V$ of $\left(A, \tau_{\rho}\right)$ such that $[a]_{\equiv_{\rho}}=U \cup V$. If $u \in U, v \in V$, then $u \equiv \equiv_{\rho} v \equiv_{\rho} a$. By (1), it follows that $[a]_{\equiv_{\rho}} \subseteq U \cap V$, proving that $[a]_{\equiv_{\rho}}$ is connected. 
(3) Let $B$ be a clopen subset of $A$. By (1), it is clear that $B=\bigcup_{a \in B}[a]_{\equiv_{\rho}}$.

(4) By (2), every equivalence class modulo $\equiv_{\rho}$ is a connected component of $A$, since it is connected and clopen. Conversely, let $Y$ be a connected component of $A$. Note that, for every $a \in Y,[a]_{\equiv \rho_{\rho}} \cap Y$ is clopen in $Y$ relatively to the subspace topology. Since $Y=\bigcup_{a \in Y}\left([a]_{\equiv{ }_{\rho}} \cap\right.$ $Y)$ and every union of clopen subsets is clopen because $\left(A, \tau_{\rho}\right)$ is an Alexandroff-discrete space, the fact that $Y$ is connected implies that there exists an element $a_{0} \in Y$ such that $Y=\left[a_{0}\right]_{\equiv_{\rho}} \cap Y$. In other words, $Y \subseteq\left[a_{0}\right]_{\equiv_{\rho}}$, and since $Y$ is a connected component and $\left[a_{0}\right]_{\equiv_{\rho}}$ is connected, we infer that $\left[a_{0}\right]_{\equiv_{\rho}}=Y$.

An immediate consequence of Lemmas 2.8 and 2.9 is that

$$
A=\bigcup_{[a]_{\equiv \rho} \in A / \equiv \rho}[a]_{\equiv \rho}
$$

is the unique coproduct decomposition of a preordered set $(A, \rho)$ into a disjoint union of indecomposable clopen subsets. That is, every Alexandroff-discrete space is the coproduct of its connected components.

\section{The stable category}

We will now construct from Preord a pointed quotient category in which one may consider the key instruments of homological algebra, like short exact sequences (Sect. 3). For every pair of objects $(A, \rho),\left(A^{\prime}, \rho^{\prime}\right)$ in Preord, let $R_{A, A^{\prime}}$ be the relation on the $\operatorname{set} \operatorname{Hom}\left(A, A^{\prime}\right)$ defined, for every $f, g:(A, \rho) \rightarrow\left(A^{\prime}, \rho^{\prime}\right)$, by $f R_{A, A^{\prime}} g$ if there exists a clopen subset $B$ of $A$ such that $\left.f\right|_{B}$ and $\left.g\right|_{B}$ are two trivial morphisms and $\left.f\right|_{A \backslash B}=\left.g\right|_{A \backslash B}$. By definition, given arbitrary trivial morphisms $f, g: A \rightarrow A^{\prime}$, we have that $f R_{A, A^{\prime}} g$. In the next lemma, we make use of the terminology of $[9$, p. 51].

\section{Lemma 3.1 The assignment $\left(A, A^{\prime}\right) \mapsto R_{A, A^{\prime}}$ is a congruence on the category Preord.}

Proof The relations $R_{A, A^{\prime}}$ are clearly reflexive and symmetric. They are also transitive, because if $f, g, h:(A, \rho) \rightarrow\left(A^{\prime}, \rho^{\prime}\right), f R_{A, A^{\prime}} g$ and $g R_{A, A^{\prime}} h$, then there exist clopen subsets $B, C$ of $A$ such that $\left.f\right|_{B},\left.g\right|_{B},\left.g\right|_{C}$ and $\left.h\right|_{C}$ are four trivial morphisms, $\left.f\right|_{A \backslash B}=\left.g\right|_{A \backslash B}$ and $\left.g\right|_{A \backslash C}=\left.h\right|_{A \backslash C}$. Then $B \cup C$ is a clopen subset of $A$. Let us prove that $\left.f\right|_{B \cup C}$ is a trivial morphism. Suppose $x, y \in B \cup C$ and $x \rho y$. Then we have two cases: either $x, y \in B$ or $x, y \in C \backslash B$. If $x, y \in B$, then $f(x)=f(y)$ because $\left.f\right|_{B}$ is a trivial morphism. If $x, y \in C \backslash B$, then $f(x)=g(x)$ and $f(y)=g(y)$ because $\left.f\right|_{A \backslash B}=\left.g\right|_{A \backslash B}$. As $\left.g\right|_{C}$ is a trivial morphism, it follows that $g(x)=g(y)$; hence, $f(x)=f(y)$. This proves that $\left.f\right|_{B \cup C}$ is a trivial morphism. Similarly, $\left.h\right|_{B \cup C}$ is a trivial morphism. Finally, if $x \in A \backslash(B \cup C)$, then $f(x)=g(x)$ because $\left.f\right|_{A \backslash B}=\left.g\right|_{A \backslash B}$ and $g(x)=h(x)$ because $\left.g\right|_{A \backslash C}=\left.h\right|_{A \backslash C}$. Thus $f(x)=h(x)$. This proves that $\left.f\right|_{A \backslash(B \cup C)}=\left.h\right|_{A \backslash(B \cup C)}$. Therefore, $R_{A, A^{\prime}}$ is an equivalence relation on $\operatorname{Hom}\left(A, A^{\prime}\right)$ for each pair $\left(A, A^{\prime}\right)$ of objects of Preord.

Finally, suppose that $f, g:(A, \rho) \rightarrow\left(A^{\prime}, \rho^{\prime}\right)$ with $f R_{A, A^{\prime}} g, h:(B, \sigma) \rightarrow(A, \rho)$ and $\ell:\left(A^{\prime}, \rho^{\prime}\right) \rightarrow(C, \tau)$. Then there exists a clopen subset $X$ of $A$ such that $\left.f\right|_{X}$ and $\left.g\right|_{X}$ are two trivial morphisms and $\left.f\right|_{A \backslash X}=\left.g\right|_{A \backslash X}$. Then $h^{-1}(X)$ is a clopen subset of $(B, \sigma)$. The mapping $\left.\ell f h\right|_{h^{-1}(X)}$ is a trivial morphism, because if $b, b^{\prime} \in h^{-1}(X)$ and $b \sigma b^{\prime}$, then $h(b), h\left(b^{\prime}\right) \in X$ and $h(b) \rho h\left(b^{\prime}\right)$. Since $\left.f\right|_{X}$ is a trivial morphism, we get that $f h(b)=f h\left(b^{\prime}\right)$. Therefore, $\ell f h(b)=\ell f h\left(b^{\prime}\right)$. This proves that $\left.\ell f h\right|_{h^{-1}(X)}$ is a trivial morphism. Similarly, $\left.\ell g h\right|_{h^{-1}(X)}$ is a trivial morphism. It remains to prove that 
$\left.\ell f h\right|_{B \backslash h^{-1}(X)}=\left.\ell g h\right|_{B \backslash h^{-1}(X)}$. Suppose $b \in B \backslash h^{-1}(X)$. Then $h(b) \in A \backslash X$, so that $f(h(b))=g(h(b))$ because $\left.f\right|_{A \backslash X}=\left.g\right|_{A \backslash X}$. Therefore, $\ell(f(h(b)))=\ell(g(h(b)))$.

It is therefore possible to construct the quotient category Preord:=Preord $/ R$. We will call it the stable category. Its objects are all preordered sets $(A, \rho)$, like in Preord. The morphisms $(A, \rho) \rightarrow\left(A^{\prime}, \rho^{\prime}\right)$ are the equivalence classes of $\operatorname{Hom}\left(A, A^{\prime}\right)$ modulo $R_{A, A^{\prime}}$, that is,

$$
\operatorname{Hom}_{\text {Preord }}\left(A, A^{\prime}\right):=\operatorname{Hom}_{\operatorname{Preord}}\left(A, A^{\prime}\right) / R_{A, A^{\prime}} \text {. }
$$

There is a canonical functor $F:$ Preord $\rightarrow$ Preord. Our notation will be $F(A)=\underline{A}$ for every object $A$ and $F(f)=\underline{f}$ for every morphism $f: A \rightarrow A^{\prime}$.

Remark 3.2 The functor $F$ preserves coproducts. In fact, let $\left(A_{i}, \rho_{i}\right), i \in I$, be preordered sets and let $C:=\left(\coprod_{i \in I} A_{i}, \rho\right)$ be their coproduct in Preord, so $C$ is the disjoint union and $\rho$ is the coproduct preorder. For any $i \in I$, let $\varepsilon_{i}: A_{i} \rightarrow C$ be the canonical embedding (for all $\left.x \in A_{i}, \varepsilon_{i}(x):=(x, i)\right)$. Fix any preordered set $Y$, and for all $i \in I$, consider morphisms $f_{i}: A_{i} \rightarrow \underline{Y}$ in Preord. By the universal property of $C$ in Preord, there exists a unique morphism $f: C \rightarrow Y$ (in Preord) such that $f_{i}=f \circ \varepsilon_{i}$ for all $i \in I$. In particular, $\underline{f_{i}}=\underline{f} \circ \underline{\varepsilon_{i}}$. Suppose there is a morphism $\underline{g}: \underline{C} \rightarrow \underline{Y}$ in Preord such that $f_{i}=\underline{g} \circ \underline{\varepsilon_{i}}$, for $\overline{a n y} i \in I$. According to the last condition, for every $i \in I$ there exists a clopen subset $B_{i}$ of $A_{i}$ such that $\left.f_{i}\right|_{B_{i}}$ and $\left.\left(g \circ \varepsilon_{i}\right)\right|_{B_{i}}$ are trivial morphisms and $\left.f_{i}\right|_{A_{i} \backslash B_{i}}=\left.\left(g \circ \varepsilon_{i}\right)\right|_{A_{i} \backslash B_{i}}$. By Remark 2.6(2), the canonical image $B_{i} \times\{i\}$ of $B_{i}$ in $C$ is clopen in $C$, and since $\left(C, \tau_{\rho}\right)$ is Alexandroff-discrete, $B:=\bigcup_{i \in I} B_{i} \times\{i\}$ is clopen in $C$. Now it is straightforward to check that $\left.f\right|_{B},\left.g\right|_{B}$ are trivial morphisms and that $\left.f\right|_{C \backslash B}=\left.g\right|_{C \backslash B}$. Hence $\underline{f}=\underline{g}$. This concludes the proof.

For every preordered set $(A, \rho)$, let $\pi: A \rightarrow A / \equiv_{\rho}, \pi: a \mapsto[a]_{\equiv_{\rho}}$, be the canonical projection. Let $A^{*}$ be the clopen subset of $A$ defined by

$$
A^{*}:=\bigcup_{\substack{a \in A \\|\pi(a)|>1}} \pi(a) .
$$

We will say that $(A, \rho)$ is reduced if $A=A^{*}$. Therefore, every preordered set $A$ is the disjoint union, in a unique way, of two clopen sets: the reduced object $A^{*}$ and the trivial object $A \backslash A^{*}$.

Theorem 3.3 The following conditions are equivalent for two non-empty preordered sets $(A, \rho),\left(A^{\prime}, \rho^{\prime}\right)$ :

1. $(A, \rho),\left(A^{\prime}, \rho^{\prime}\right)$ are isomorphic objects in Preord.

2. $\left(A^{*}, \rho\right),\left(A^{* *}, \rho^{\prime}\right)$ are isomorphic objects in Preord.

3. There exist trivial objects $X, X^{\prime}$ in Preord such that $A \amalg X \cong A^{\prime} \bigsqcup X^{\prime}$ in Preord.

Proof First of all, we prove some preliminary steps.

Step 1. For every non-trivial preordered set $(A, \rho)$, the sets $A$ and $A^{*}$ with the induced preorder are isomorphic objects in Preord.

From $A$ non-trivial, we have $A^{*} \neq \emptyset$. Fix an element $a_{0}$ in $A^{*}$. Let $f: A \rightarrow A^{*}$ be defined by $f(x)=x$ for every $x \in A^{*}$ and $f(x)=a_{0}$ for every $x \in A \backslash A^{*}$. Let $\varepsilon: A^{*} \rightarrow A$ be the inclusion. Then $f \varepsilon$ is the identity $1_{A^{*}}$ on $A^{*}$, and $\varepsilon f R_{A, A} 1_{A}$, because (1) $\left.\varepsilon f\right|_{A^{*}}=\left.1_{A}\right|_{A^{*}}$, (2) $\left.\varepsilon f\right|_{A^{*}}$ is the constant mapping equal to $a_{0}$, hence a trivial morphism, and (3) $\left.1_{A}\right|_{A \backslash A^{*}}$ is the trivial morphism because $A \backslash A^{*}$ is a trivial object. Hence $A$ and $A^{*}$ are isomorphic in Preord. 
Step 2. If $\left(A^{*}, \rho\right)$ is a reduced preordered set and $f$ is an endomorphism of $\left(A^{*}, \rho\right)$ such that $f R_{A^{*}, A^{*}} 1_{A^{*}}$, then $f=1_{A^{*}}$.

Suppose $f R_{A^{*}, A^{*}} 1_{A^{*}}$. Then there exists a clopen subset $B$ of $A^{*}$ such that $\left.f\right|_{B}$ and $\left.1_{A^{*}}\right|_{B}$ are two trivial morphisms and $\left.f\right|_{A^{*} \backslash B}=\left.1_{A^{*}}\right|_{A^{*} \backslash B}$. But $\left.1_{A^{*}}\right|_{B}$ trivial morphism and $A^{*}$ reduced implies $B=\emptyset$. Thus $f=1_{A^{*}}$ follows from $\left.f\right|_{A^{*} \backslash B}=\left.1_{A^{*}}\right|_{A^{*} \backslash B}$.

Step 3. Two reduced preordered sets $\left(A^{*}, \rho\right),\left(A^{\prime *}, \rho^{\prime}\right)$ are isomorphic objects in Preord if and only if they are isomorphic objects in Preord.

Assume $\left(A^{*}, \rho\right)$ and $\left(A^{\prime *}, \rho^{\prime}\right)$ isomorphic in Preord. Then there exist morphisms $f: A^{*} \rightarrow A^{\prime *}$ and $g: A^{\prime *} \rightarrow A^{*}$ in Preord such that $g f R_{A^{*}, A^{*}} 1_{A^{*}}$ and $f g R_{A^{\prime *}, A^{* *}} 1_{A^{\prime *}}$. By Step 2, we have $g f=1_{A^{*}}$ and $f g=1_{A^{\prime *}}$. Thus $\left(A^{*}, \rho\right)$ and $\left(A^{\prime *}, \rho^{\prime}\right)$ are isomorphic in Preord. The converse is clear.

Step 4. If $(A, \rho),\left(A^{\prime}, \rho^{\prime}\right)$ are non-empty preordered sets that are isomorphic in Preord, then $(A, \rho)$ is trivial if and only if $\left(A^{\prime}, \rho^{\prime}\right)$ is trivial.

Suppose $(A, \rho)$ trivial. By definition, there are morphisms $f:\left(A^{\prime}, \rho^{\prime}\right) \rightarrow(A, \rho), g$ : $(A, \rho) \rightarrow\left(A^{\prime}, \rho^{\prime}\right)$ such that $g f=1_{A^{\prime}}$. Hence there is a clopen subset $B \subseteq A^{\prime}$ with $\left.1_{A^{\prime}}\right|_{B}$ trivial and $\left.g f\right|_{A^{\prime} \backslash B}=\left.1_{A^{\prime}}\right|_{A^{\prime} \backslash B}$. Take $x, y \in A^{\prime}$ such that $x \rho^{\prime} y$. If $x, y \in B$, the fact that $\left.1_{A^{\prime}}\right|_{B}$ is trivial implies $x=y$. Otherwise, we must have $x, y \in A^{\prime} \backslash B$, since $B$ is clopen. Since $(A, \rho)$ is trivial and $\left.g f\right|_{A^{\prime} \backslash B}=\left.1_{A^{\prime}}\right|_{A^{\prime} \backslash B}$, it immediately follows $x=y$.

Step 5. If $A$ is a non-empty preordered set and $X$ is a trivial object in Preord, then $A$ and $A \amalg X$ (the coproduct in Preord) are isomorphic objects in $\underline{\text { Preord. }}$.

Two mutually inverse isomorphisms in Preord are induced: (1) by the coproduct embed$\operatorname{ding} A \rightarrow A \amalg X$ and (2) by the coproduct $A \amalg X \rightarrow A$ of the identity mapping $1_{A}$ of $A$ and any constant mapping $X \rightarrow A$.

We are now ready to prove the equivalence of the three conditions in the statement of the theorem.

(1) $\Rightarrow(2)$. If $A, A^{\prime}$ are isomorphic in Preord, they are either both trivial or both non-trivial by Step 4 . In the first case, $A^{*}=A^{*}=\emptyset$. In the second case, the conclusion follows from Steps 1 and 3 .

$(2) \Rightarrow(3)$. If $\left(A^{*}, \rho\right),\left(A^{* *}, \rho^{\prime}\right)$ are isomorphic in Preord, it suffices to take $X:=A^{\prime} \backslash A^{\prime *}$ and $X^{\prime}:=A \backslash A^{*}$, and (3) holds.

(3) $\Rightarrow(1)$. Assume $A \coprod X \cong A^{\prime} \bigsqcup X^{\prime}$ in Preord with $X, X^{\prime}$ trivial objects. If $A$ is trivial, then $A^{\prime}$ is also trivial, and trivial non-empty objects are isomorphic in Preord. If $A$ is nontrivial, then $A^{\prime}$ is also non-trivial, so $A \cong A \amalg X \cong A^{\prime} \amalg X^{\prime} \cong A^{\prime}$ in Preord by Step 5 .

Remark 3.4 It is worth noting that the assumption that $A, A^{\prime}$ are non-empty in the statement of Theorem 3.3 is necessary. Indeed, if $(A,=)$ is a non-empty trivial object Preord and $A^{\prime}=\emptyset$, then $(A,=), A^{\prime}$ are obviously not isomorphic in Preord, but $A^{*}=A^{*}=\emptyset$.

Definition 3.5 Let $f: A \rightarrow A^{\prime}$ be a morphism in Preord. We say that a morphism $k: X \rightarrow A$ in Preord is a prekernel of $f$ if the following properties are satisfied:

1. $f k$ is a trivial morphism.

2. Whenever $\lambda: Y \rightarrow A$ is a morphism in Preord and $f \lambda$ is trivial, then there exists a unique morphism $\lambda^{\prime}: Y \rightarrow X$ in Preord such that $\lambda=k \lambda^{\prime}$.

Recall that, for every mapping $f: A \rightarrow A^{\prime}$, the equivalence relation $\sim_{f}$ on $A$, associated with $f$, is defined, for every $a, b \in A$, by $a \sim_{f} b$ if $f(a)=f(b)$. It is easy to show that every morphism in Preord has a prekernel. More precisely: 
Proposition 3.6 Let $f:(A, \rho) \rightarrow\left(A^{\prime}, \rho^{\prime}\right)$ be a morphism in Preord. Then a prekernel of $f$ is the morphism $k:\left(A, \rho \cap \sim_{f}\right) \rightarrow(A, \rho)$, where $k$ is the identity mapping and $\sim_{f}$ is the equivalence relation on $A$ associated with $f$.

We do not give a proof of the next proposition here, because it will be proved in a more general form in Proposition 5.1.

Proposition 3.7 Let $f: A \rightarrow A^{\prime}$ be a morphism in Preord, and let $\mu: X \rightarrow$ A be a prekernel of $f$. The following properties hold.

1. $\mu$ is a monomorphism.

2. If $\lambda: Y \rightarrow A$ is any other prekernel of $f$, then there exists a unique isomorphism $\lambda^{\prime}: Y \rightarrow X$ such that $\lambda=\mu \lambda^{\prime}$.

The category Preord has an initial object (the empty set) and terminal objects (the preordered sets with one element). Thus initial objects are different from terminal objects in Preord. Hence the same is true in its quotient category Preord:=Preord $/ R$. In order to define kernels, cokernels and exact sequences, we need a pointed category, and we have just seen that Preord is not a pointed category. But to get a pointed category, it is sufficient to eliminate the empty set, i.e. consider the full subcategory Preord* of Preord whose objects are all $(A, \rho)$ with $A \neq \emptyset$ and the full subcategory $\underline{\text { Preord }}^{*}$ of the category Preord whose objects are all objects of Preord except for the empty set. More precisely, the category $\underline{\text { Preord }}^{*}$ is a pointed category because its zero object is $\underline{0}=\underline{T}$ for every non-empty trivial object $(T,=)$. For any pair of objects $\underline{A}, \underline{A}^{\prime}$ of Preord ${ }^{*}$, it is possible therefore to define the zero morphism $\underline{0}_{\hat{A}, \underline{A}^{\prime}}: \underline{A} \rightarrow \underline{A^{\prime}}$ as the composite morphism of the unique morphism of $\underline{A}$ into $\underline{0}$ and the unique morphism of $\underline{0}$ into $\underline{A^{\prime}}$. For instance, for any constant mapping $c: A \rightarrow A^{\prime}$, we find that $\underline{c}: \underline{A} \rightarrow \underline{A^{\prime}}$ is clearly $\underline{0}_{A}, \underline{A^{\prime}}: \underline{A} \rightarrow \underline{A^{\prime}}$. More generally, for a morphism $g: A \rightarrow A^{\prime}$, one has that $\underline{g}=\underline{0}_{\underline{A}}, \underline{A}^{\prime}$ if and only if $g$ is a trivial morphism. By definition, the kernel of any morphism $\underline{f}: \underline{A} \rightarrow \underline{A}^{\prime}$ in $\underline{\text { Preord }}^{*}$ is the equalizer of $\underline{f}$ and the zero morphism $\underline{0}_{\hat{A}, \underline{A}^{\prime}}$.

Remark 3.8 By Theorem 3.3, the situation for the category Preord* is extremely similar to the case of the category mod- $R$ of finitely presented right modules over a semiperfect ring $R$ [10, Introduction and Theorem 1.4]. In both cases, every object is, in a unique way, a coproduct of a projective object and an object with no nonzero projective direct summands, we factor out modulo projective objects, two objects are isomorphic in the stable category if and only if they are stably isomorphic (recall that $A$ and $B$ are stably isomorphic if there are projective $P$ and $Q$ such that $A \oplus P \cong B \oplus Q$ ), every stable isomorphism class of finitely generated modules over a semiperfect ring contains a unique minimal element (up to isomorphism) and so on.

Proposition 3.9 If $f:(A, \rho) \rightarrow\left(A^{\prime}, \rho^{\prime}\right)$ is a morphism in Preord with $A, A^{\prime} \neq \varnothing$ and $k:\left(A, \rho \cap \sim_{f}\right) \rightarrow(A, \rho)$ is a prekernel of $f$, then $\underline{k}$ is a kernel of $\underline{f}$ in the pointed category Preord $^{*}$.

Proof We must show that $\underline{k}$ satisfies the universal property of kernels. Since $f k$ is trivial (see Proposition 3.6), the morphism $f k$ is the zero morphism. Take any morphism $k^{\prime}:\left(K^{\prime}, \tau\right) \rightarrow$ $(A, \rho)$ in Preord* such that $f k^{\prime}$ is the zero morphism, i.e. $f k^{\prime}$ is trivial. By Proposition 3.6, there exists a unique morphism $u:\left(K^{\prime}, \tau\right) \rightarrow\left(A, \rho \cap \sim_{f}\right)$ such that $k^{\prime}=k u$. In particular, $\underline{k}^{\prime}=\underline{k u}$. For the uniqueness of $\underline{u}$ in $\underline{\text { Preord }}^{*}$, we have that the identity $A \rightarrow A$ is another prekernel by Proposition 3.6, so that $k$ is an isomorphism by Proposition 3.7(2). 
Example 3.10 (Quotient preordered set) Let $(A, \rho)$ be a preordered set and $\sim$ an equivalence relation on $A$. Then $\rho$ induces a well-defined preorder $\rho^{\prime}$ on the quotient set $A / \sim$ (via the position $\left[a_{1}\right] \sim \rho^{\prime}\left[a_{2}\right] \sim$ if $a_{1} \rho a_{2}$ for all $a_{1}, a_{2} \in A$ ) if and only if $\sim \subseteq \rho$. In fact, if the relation $\rho^{\prime}$ on the quotient set $A / \sim$ is a well-defined reflexive relation, then $a_{1} \sim a_{2}$ implies $\left[a_{1}\right] \sim=\left[a_{2}\right] \sim$, so $\left[a_{1}\right] \sim \rho^{\prime}\left[a_{2}\right] \sim$, i.e. $a_{1} \rho a_{2}$. This proves that $\sim \subseteq \rho$. Conversely, if $\sim \subseteq \rho$, then $\left[a_{1}\right]_{\sim}=\left[a_{2}\right]_{\sim}$ and $\left[a_{3}\right]_{\sim}=\left[a_{4}\right] \sim$ imply $a_{1} \rho a_{3}$ if and only if $a_{2} \rho a_{4}$.

If these two equivalent conditions hold, then the canonical projection $\pi: A \rightarrow A / \sim$ is a morphism in Preord and its prekernel is the identity $k:(A, \sim) \rightarrow(A, \rho)$.

For simplicity of notation, we will indicate by the same symbol $\rho$ the preorder induced by $\rho$ on the quotient set $A / \sim$, provided $\sim \subseteq \rho$.

Remark 3.11 Let $A$ be a set and let $\sim, \rho$ be, respectively, an equivalence relation and a preorder on $A$ such that $\sim \subseteq \rho$. As we have seen, the canonical projection $\pi:(A, \rho) \rightarrow$ $(A / \sim, \rho)$ is a morphism in Preord. Then:

1. If $B \subseteq A$ is a clopen set, then $\pi(B)$ is clopen in $Q:=A / \sim$.

2. $\underline{\pi}$ is an epimorphism in Preord.

Proof (1) Take elements $p \in \pi(B)$ and $q \in Q \backslash \pi(B)$, thus $p=\pi(b), q=\pi(x)$ for some $b \in B, x \in A \backslash B$. Since $B$ is clopen in $A$, we have $b \not \varnothing x$ and $x \not \varnothing b$ and, by definition, $p \not \supset q$ and $q \not \supset p$.

(2) Let $g, h:(Q, \rho) \rightarrow(T, \tau)$ be morphisms in Preord with $g \pi=\underline{h \pi}$ in Preord. By definition, there is a clopen set $B \subseteq A$ such that $\left.(g \pi)\right|_{B},\left.(h \pi)\right|_{B} \overline{\text { are trivial morphisms and }}$ $\left.(g \pi)\right|_{A \backslash B}=\left.(h \pi)\right|_{A \backslash B}$. By part (1), $B^{\prime}:=\pi(B)$ is clopen in $Q$ and it is easily seen that $\pi(A \backslash B)=Q \backslash B^{\prime}$. Then $\left.g\right|_{B^{\prime}},\left.h\right|_{B^{\prime}}$ are trivial morphisms and $\left.g\right|_{Q \backslash B^{\prime}}=\left.g\right|_{Q \backslash B^{\prime}}$. Hence $\underline{g}=\underline{h}$, and the conclusion follows.

Definition 3.12 Let $f: A \rightarrow A^{\prime}$ be a morphism in Preord. A precokernel of $f$ is a morphism $p: A^{\prime} \rightarrow X$ such that:

1. $p f$ is a trivial map.

2. Whenever $\lambda: A^{\prime} \rightarrow Y$ is a morphism such that $\lambda f$ is trivial, then there exists a unique morphism $\lambda_{1}: X \rightarrow Y$ with $\lambda=\lambda_{1} p$.

If $f:(A, \rho) \rightarrow\left(A^{\prime}, \rho^{\prime}\right)$ is a morphism in Preord, consider the canonical projection $c:\left(A^{\prime}, \rho^{\prime}\right) \rightarrow\left(A^{\prime} / \zeta_{f}, \rho^{\prime} \vee \zeta_{f}\right)$, where $\zeta_{f}$ is the equivalence relation on $A^{\prime}$ generated by the set $\left\{\left(f\left(a_{1}\right), f\left(a_{2}\right)\right) \mid a_{1}, a_{2} \in A, a_{1} \rho a_{2}\right\}$ and $\vee$ is the least upper bound in the complete lattice of all preorders on the set $A^{\prime}$ (that is, $\rho^{\prime} \vee \zeta_{f}$ is the preorder on $A^{\prime}$ generated by $\rho^{\prime}$ and $\left.\zeta_{f}\right)$. Thus $\zeta_{f}$ is the transitive closure of the relation $\left\{\left(a^{\prime}, a^{\prime}\right) \mid a^{\prime} \in A^{\prime}\right\} \cup\left\{\left(f\left(a_{1}\right), f\left(a_{2}\right)\right) \mid\right.$ $\left.a_{1}, a_{2} \in A, a_{1} \sim_{\rho} a_{2}\right\}$. Since the equivalence relation $\zeta_{f}$ is contained in the preorder $\rho^{\prime} \vee \zeta_{f}$, it is possible to construct the quotient preordered set (Example 3.10), and thus, $\rho^{\prime} \vee \zeta_{f}$ induces a preorder on the quotient set $A^{\prime} / \zeta_{f}$ (which will be still denoted by $\rho^{\prime} \vee \zeta_{f}$, with a small abuse of notation).

Proposition 3.13 Let $f:(A, \rho) \rightarrow\left(A^{\prime}, \rho^{\prime}\right)$ be a morphism in Preord. Then the canonical projection $c:\left(A^{\prime}, \rho^{\prime}\right) \rightarrow\left(A^{\prime} / \zeta_{f}, \rho^{\prime} \vee \zeta_{f}\right)$ is a precokernel of $f$.

Proof We must prove that $c f$ is a trivial morphism and that, for every morphism $g:\left(A^{\prime}, \rho^{\prime}\right) \rightarrow$ $(B, \sigma)$ with $g f$ a trivial morphism, there exists a unique morphism $g^{\prime}:\left(A^{\prime} / \zeta_{f}, \rho^{\prime} \vee \zeta_{f}\right) \rightarrow$ $(B, \sigma)$ such that $g=g^{\prime} c$. If $x, y \in A$ and $x \rho y$, then $f(x) \zeta_{f} f(y)$ by the definition of $\zeta_{f}$. So $c f(x)=c f(y)$. This shows that $c f$ is a trivial morphism. Now let $g:\left(A^{\prime}, \rho^{\prime}\right) \rightarrow(B, \sigma)$ 
be a morphism in Preord with $g f$ a trivial morphism. If $x, y \in A$ and $x \rho y$, we get that $g f(x)=g f(y)$. It follows that $\zeta_{f} \subseteq \sim_{g}$. This shows that the position $\left[a^{\prime}\right]_{\zeta_{f}} \mapsto g(a)$ defines a mapping $g^{\prime}: A^{\prime} / \zeta_{f} \rightarrow B$, and clearly $g$ factors uniquely through the canonical projection $c$ and the mapping $g^{\prime}$. The mapping $g^{\prime}$ is a morphism $\left(A^{\prime} / \zeta_{f}, \rho^{\prime} \vee \zeta_{f}\right) \rightarrow(B, \sigma)$ in Preord. To see this, consider elements $[a]_{\zeta_{f}},[\alpha]_{\zeta_{f}}$ such that $a\left(\zeta_{f} \vee \rho^{\prime}\right) \alpha$. By definition, there are elements $x_{0}:=a, x_{1}, \ldots, x_{n}:=\alpha \in A^{\prime}$ such that, for every $0 \leq i \leq n-1$, either $x_{i} \zeta_{f} x_{i+1}$ or $x_{i} \rho^{\prime} x_{i+1}$. In the first case, since $\zeta_{f} \subseteq \sim_{g}$, it follows that $g^{\prime}\left(x_{i}\right)=g^{\prime}\left(x_{i+1}\right)$. In the second case, since $g$ is a morphism, $g\left(x_{i}\right) \sigma g\left(x_{i+1}\right)$. Thus in both cases, we have $g^{\prime}\left(\left[x_{i}\right]_{\zeta_{f}}\right)=g\left(x_{i}\right) \sigma g\left(x_{i+1}\right)=$ $g^{\prime}\left(\left[x_{i+1}\right]_{\zeta_{f}}\right)$. Hence $g^{\prime}\left([a]_{\zeta_{f}}\right) \sigma g^{\prime}\left([\alpha]_{\zeta_{f}}\right)$, because $\sigma$ is transitive.

It is not difficult to prove the next result directly, but it will also be an immediate consequence of our more general Proposition 5.2.

Proposition 3.14 Let $f: A \rightarrow A^{\prime}$ be a morphism in Preord.

1. Every precokernel of $f$ is an epimorphism.

2. If $p: A^{\prime} \rightarrow X, q: A^{\prime} \rightarrow Y$ are cokernels of $f$, then there exists a unique isomorphism $\varphi: X \rightarrow Y$ such that $q=\varphi p$.

Proposition 3.15 Let $f:(A, \rho) \rightarrow(B, \sigma)$ be a morphism in Preord and let $\pi:(B, \sigma) \rightarrow$ $\left(B / \zeta_{f}, \sigma \vee \zeta_{f}\right)$ be its precokernel. Then

1. $\zeta_{f} \subseteq \equiv_{\sigma}$, where $\equiv_{\sigma}$ denotes the equivalence relation generated by $\sigma$.

2. If $C \subseteq B$ is a clopen set, then $\pi(C) \subseteq B / \zeta_{f}$ is a clopen set.

3. $\pi$ is an epimorphism in Preord.

Proof (1) By definition, $\zeta_{f}$ is generated by

$$
G:=\left\{\left(f\left(a_{1}\right), f\left(a_{2}\right)\right) \mid a_{1}, a_{2} \in A, a_{1} \rho a_{2}\right\},
$$

and thus, $G \subseteq \sigma \subseteq \equiv_{\sigma}$, because $f$ is a morphism in Preord. This allows to conclude the proof.

(2) Set $Q:=B / \zeta_{f}$ and consider elements $\eta \in \pi(C)$ and $\lambda \in Q \backslash \pi(C)$. Then $\eta=\pi(c)$ and $\lambda=\pi(b)$ for some $c \in C, b \in B \backslash C$. If $\eta\left(\sigma \vee \zeta_{f}\right) \lambda$, by definition $c\left(\sigma \vee \zeta_{f}\right) b$. Thus there are elements $x_{0}:=c, x_{1}, \ldots, x_{n}=b \in B$ such that, for $0 \leq i<n$, either $x_{i} \sigma x_{i+1}$ or $x_{i} \zeta_{f} x_{i+1}$. For $i=0$, we have either $c \sigma x_{1}$ or $c \zeta_{f} x_{1}$. In the first case, we infer $x_{1} \in C$, since $C$ is clopen and $c \in C$. In the second case, there are elements $y_{0}:=c, y_{1}, \ldots, y_{m}:=x_{1} \in B$ such that, for $0 \leq j<m$, either $y_{j} \sigma y_{j+1}$ or $y_{j+1} \sigma y_{j}$. In particular, since $c \sigma y_{1}$ or $y_{1} \sigma c$ and $C$ is clopen, it follows that $y_{1} \in C$. By induction on $j$, we have $y_{m}=x_{1} \in C$. This proves that in both cases $c \sigma x_{1}$ and $c \zeta_{f} x_{1}$, we have $x_{1} \in C$. By induction on $i$, we get $x_{n}=b \in C$, a contradiction.

(3) Argue as in Remark 3.11(2), noting that if $C$ is a clopen subset of $B$, then $\pi(B \backslash C)=$ $Q \backslash \pi(C)$.

Proposition 3.16 Let $f:(A, \rho) \rightarrow(B, \sigma)$ be a morphism in Preord*, and let

$$
\pi:(B, \sigma) \rightarrow\left(B / \zeta_{f}, \sigma \vee \zeta_{f}\right)
$$

be a precokernel of $f$. Then $\underline{\pi}$ is a cokernel of $\underline{f}$ in the pointed category Preord $^{*}$.

Proof By Proposition 3.13, $\pi f$ is trivial, that is, $\pi f=0$ in Preord ${ }^{*}$. Now, let $g:(B, \sigma) \rightarrow$ $(C, \tau)$ be a morphism in Preord such that $g f \overline{=} 0$ in $\underline{\text { Preord }}^{*}$, that is, $g f$ is trivial. By Proposition 3.13 again, there exists a unique morphism $g^{\prime}:\left(B / \zeta_{f}, \sigma \vee \zeta_{f}\right) \rightarrow(C, \tau)$ in 
Preord such that $g=g^{\prime} \pi$, so $\underline{g}=g^{\prime} \pi$. The uniqueness of such a $\underline{g}^{\prime}$ in $\underline{\text { Preord }}^{*}$ follows from the fact that $\underline{\pi}$ is an epimorphism in Preord, in view of Propositions 3.13, 3.14(1) and $3.15(3)$.

Definition 3.17 Let $f: X \rightarrow Y$ and $g: Y \rightarrow Z$ be morphisms in Preord. We say that $X \stackrel{f}{\longrightarrow} Y \stackrel{g}{\longrightarrow} Z$ is a short preexact sequence in Preord if $f$ is a prekernel of $g$ and $g$ is a precokernel of $f$.

Example 3.18 Let $A$ be any set, let $\rho$ be a preorder on $A$ and let $\sim$ be an equivalence relation on $A$ such that $\sim \subseteq \rho$. By Example 3.10, $\rho$ induces a preorder on the quotient set $A / \sim$, which we also denote by $\rho$. Then

$$
(A, \sim) \stackrel{k}{\longrightarrow}(A, \rho) \stackrel{\pi}{\longrightarrow}(A / \sim, \rho)
$$

is a short preexact sequence in Preord, where $k$ is the identity map and $\pi$ is the canonical projection. The equalities $\sim_{\pi} \cap \rho=\sim \cap \rho=\sim$ and Proposition 3.6 imply that $k$ is a prekernel of $\pi$. Moreover, by definition $\zeta_{k}=\sim$ and $\rho \vee \zeta_{k}=\rho \vee \sim=\rho$; hence, $\pi$ is a precokernel of $k$ by Proposition 3.13.

In particular, if $\simeq{ }_{\rho}$ is the equivalence relation on $A$ defined by $a \simeq{ }_{\rho} b$ if $a \rho b$ and $b \rho a$ (so that in particular we have $\simeq{ }_{\rho} \subseteq \rho$ ) and $\leq_{\rho}$ is the partial order on $A / \simeq_{\rho}$ induced by $\rho$ (see Proposition 2.2), then

$$
(A, \simeq \rho) \stackrel{k}{\longrightarrow}(A, \rho) \stackrel{\pi}{\longrightarrow}\left(A / \simeq_{\rho}, \leq_{\rho}\right)
$$

is a short preexact sequence in Preord.

Example 3.19 Let $f:(A, \rho) \rightarrow B$ be a morphism in Preord. Consider the canonical prekernel $k:\left(A, \rho \cap \sim_{f}\right) \rightarrow(A, \rho)$ of $f$ (Proposition 3.6). Let $\pi:(A, \rho) \rightarrow\left(A / \zeta_{k}, \rho \vee \zeta_{k}\right)$ be the canonical precokernel of $k$, according to Proposition 3.13. Then

$$
\left(A, \rho \cap \sim_{f}\right) \stackrel{k}{\longrightarrow}(A, \rho) \stackrel{\pi}{\longrightarrow}\left(A / \zeta_{k}, \rho \vee \zeta_{k}\right)
$$

is a short preexact sequence in Preord. To prove it, we only need to show that $k$ is a prekernel of $\pi$. This follows from the equalities $\rho \cap \sim_{\pi}=\rho \cap \zeta_{k}=\rho \cap \sim_{f}$ and Proposition 3.6.

In the next proposition, we determine all short preexact sequences in Preord, up to isomorphism.

Proposition 3.20 Let $(X, \rho) \stackrel{f}{\longrightarrow}(Y, \sigma) \stackrel{g}{\longrightarrow}(Z, \tau)$ be any short preexact sequence in Preord. Then there exists a commutative diagram

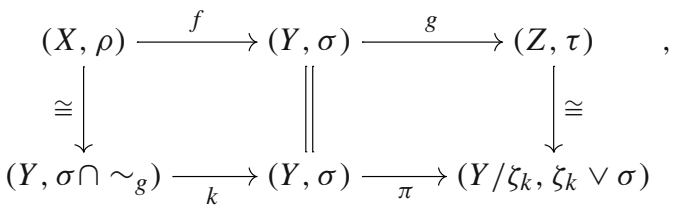

where $k$ is the identity map, $\pi$ is the canonical projection and

$$
\left(Y, \sigma \cap \sim_{g}\right) \stackrel{k}{\longrightarrow}(Y, \sigma) \stackrel{\pi}{\longrightarrow}\left(Y / \zeta_{k}, \zeta_{k} \vee \sigma\right)
$$

is a short preexact sequence. 
Proof By Proposition 3.6, $k$ is a prekernel of $g$. Since, by assumption, $f$ is also a prekernel for $g$, we infer from Proposition 3.7(2) the existence of an isomorphism $(X, \rho) \rightarrow\left(Y, \sigma \cap \sim_{g}\right)$ which makes the square on the left in diagram $(*)$ commute. Now $g$ is a precokernel of $f$, so that the existence of an isomorphism $(Z, \tau) \rightarrow\left(Y / \zeta_{k}, \zeta_{k} \vee \sigma\right)$ making the right square of the diagram commute will follow by showing that $\pi$ is a precokernel of $f$ (Proposition 3.14(2)). First, we prove that $\pi f$ is trivial. Fix elements $x, y \in X$ with $x \rho y$. Since $g$ is a precokernel of $f, g f$ is trivial, and thus, $g(f(x))=g(f(y))$, that is, $f(x) \sim_{g} f(y)$. Moreover $f(x) \sigma f(y)$, because $f$ is a morphism. Since, by definition, $\zeta_{k}$ is generated by $\left\{\left(y_{1}, y_{2}\right) \in Y \times Y \mid y_{1}\left(\sigma \cap \sim_{g}\right) y_{2}\right\}$, it follows that $f(x) \zeta_{k} f(y)$, that is, $\pi f(x)=\pi f(y)$. This proves that $\pi f$ is trivial. Now, let $\lambda:(Y, \sigma) \rightarrow(T, \eta)$ be a morphism with $\lambda f$ trivial. We have to show that there exists a unique morphism $\tilde{\lambda}:\left(Y / \zeta_{k}, \zeta_{k} \vee \sigma\right) \rightarrow(T, \eta)$ such that $\lambda=\tilde{\lambda} \pi$. It is enough to show the existence of such a $\tilde{\lambda}$, the uniqueness being a trivial consequence of the fact that $\pi$ is surjective. But, since $g$ is a precokernel of $f$ and $\lambda f$ is trivial, there exists a unique morphism $\lambda_{1}:(Z, \tau) \rightarrow(T, \eta)$ such that $\lambda=\lambda_{1} g$. Now $\zeta_{k}$ is the equivalence relation generated by $\sigma \cap \sim_{g}$ and $\sim_{g}$ is an equivalence relation, so $\zeta_{k} \subseteq \sim_{g}$. Thus $\lambda_{1}$ induces a well-defined mapping $\tilde{\lambda}:\left(Y / \zeta_{k}, \zeta_{k} \vee \sigma\right) \rightarrow(T, \eta),[y]_{\zeta_{k}} \mapsto \lambda_{1}(g(y))$ and $\lambda_{1}$ clearly satisfies $\lambda=\tilde{\lambda} \pi$. We claim that $\lambda_{1}$ is a morphism. In order to see this, take $[y]_{\zeta_{k}},[z]_{\zeta_{k}} \in Y / \zeta_{k}$ with $y\left(\sigma \vee \zeta_{k}\right) z$, and let $\beta_{1}:=y, \beta_{2}, \ldots, \beta_{n}:=z \in Y$ be such that, for every $1 \leq i<n$, either $\beta_{i} \sigma \beta_{i+1}$ or $\beta_{i} \zeta_{k} \beta_{i+1}$. In the first case, we infer $g\left(\beta_{i}\right) \tau g\left(\beta_{i+1}\right)$, and thus, $\tilde{\tilde{\lambda}}\left(\left[\beta_{i}\right]_{\zeta_{k}}\right) \eta \tilde{\lambda}\left(\left[\beta_{i+1}\right]_{\zeta_{k}}\right)$, because $g, \lambda_{1}$ are morphisms. In the second case, we have, by definition, $\left[\beta_{i}\right]_{\zeta_{k}}=\left[\beta_{i+1}\right]_{\zeta_{k}}$. Thus in both cases we get $\tilde{\lambda}\left(\left[\beta_{i}\right]_{\zeta_{k}}\right) \eta \tilde{\lambda}\left(\left[\beta_{i+1}\right]_{\zeta_{k}}\right)$ for any $1 \leq i<n$. Since $\eta$ is transitive, it follows that $\tilde{\lambda}\left([y]_{\zeta_{k}}\right) \eta \tilde{\lambda}\left([z]_{\zeta_{k}}\right)$, proving that $\tilde{\lambda}$ is a morphism.

Finally, $\left(Y, \sigma \cap \sim_{g}\right) \stackrel{k}{\longrightarrow}(Y, \sigma) \stackrel{\pi}{\longrightarrow}\left(Y / \zeta_{k}, \zeta_{k} \vee \sigma\right)$ is a short preexact sequence as we saw in Example 3.19.

\section{Short exact sequences}

In a pointed category, a short exact sequence is a pair of morphisms $f: A \rightarrow B, g: B \rightarrow$ $C$ such that $f$ is a kernel of $g$ and $g$ is a cokernel of $f$. As usual, the notation will be $0 \longrightarrow A \stackrel{f}{\longrightarrow} B \stackrel{g}{\longrightarrow} C \longrightarrow 0$. We will now describe all short exact sequences in the pointed category Preord ${ }^{*}$ up to isomorphism (similarly to the description of any short exact sequence in the category Mod- $R$ of right modules over a ring $R$, which is isomorphic to a short exact sequence of the form $0 \longrightarrow A_{R} \longrightarrow B_{R} \longrightarrow A / B \longrightarrow 0$ for suitable modules $A_{R} \leq B_{R}$ ).

Proposition 4.1 For every short exact sequence

$$
0 \longrightarrow \underline{A} \stackrel{\underline{f}}{\longrightarrow} \underline{(B, \rho)} \stackrel{\underline{g}}{\longrightarrow} \underline{C} \longrightarrow 0
$$

in Preord $^{*}$, there is a commutative diagram

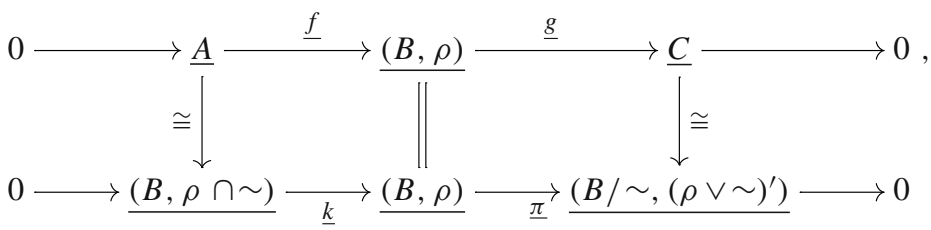


where $\sim$ is an equivalence relation on $B, k$ is the identity and $\pi$ is the canonical projection.

Proof Let $f: A \rightarrow(B, \rho), g:(B, \rho) \rightarrow C$ be representatives of $\underline{f}, \underline{g}$. Consider the sequence of morphisms

$$
\left(B, \rho \cap \sim_{g}\right) \stackrel{k}{\longrightarrow}(B, \rho) \stackrel{\pi}{\longrightarrow}\left(B / \zeta_{k}, \zeta_{k} \vee \rho\right)
$$

in Preord*, where $k$ is the identity and $\pi$ is the canonical projection. By Propositions 3.6 and 3.13, $k$ is a prekernel of $g$ and $\pi$ is a precokernel of $k$. Moreover the pair of morphisms $k, \pi$ is a short preexact sequence in Preord by Example 3.19. By Propositions 3.9 and 3.16, the canonical image

$$
\underline{\left(B, \rho \cap \sim_{g}\right)} \stackrel{\underline{k}}{\longrightarrow} \underline{(B, \rho)} \stackrel{\underline{\pi}}{\longrightarrow} \underline{\left(B / \zeta_{k}, \zeta_{k} \vee \rho\right)}
$$

is an exact sequence in the stable category Preord $^{*}$. Since $f, \underline{k}$ are kernels of $g$, there is a unique isomorphism $\underline{A} \rightarrow\left(B, \rho \cap \sim_{g}\right)$ in Preord* which makes the square on the left of diagram (**) commute. In order to get an isomorphism making the square on the right commute, it will suffice to show that $\pi$ is a cokernel of $f$. Let $\tau$ be the preorder on $A$ and let $a, b \in A$ be such that $a \tau b$. Since $f, g$ form a short exact sequence, we have, by definition, $g f=0$, i.e. $g f$ is trivial. Thus $g f(a)=g f(b)$, and since $f$ is a morphism, $f(a) \rho f(b)$, i.e. $\overline{f(a})\left(\rho \cap \sim_{g}\right) f(b)$. Since, by definition, $\zeta_{k}$ is the equivalence relation generated by $\rho \cap \sim_{g}$, it follows $[f(a)]_{\zeta_{k}}=[f(b)]_{\zeta_{k}}$, that is, $\pi f(a)=\pi f(b)$, and this proves that $\pi f$ is trivial, i.e. $\pi f=0$. Now take any morphism $\underline{\lambda}: \underline{B} \rightarrow \underline{T}$ in $\underline{\text { Preord }}^{*}$ satisfying $\underline{\lambda} f=0$. Let $\lambda: B \rightarrow T$

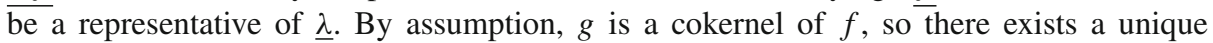
morphism $\underline{\lambda}_{1}: \underline{C} \rightarrow \underline{T}$ in $\underline{\text { Preord }}^{*}$ with $\underline{\lambda}=\underline{\lambda}_{1} g$. Let $\lambda_{1}: C \rightarrow \bar{T}$ be a representative of $\underline{\lambda_{1}}$. By definition, $\zeta_{k} \subseteq \sim_{g}$, and thus, there is a well-defined mapping $\tilde{\lambda}:\left(B / \zeta_{k}, \zeta_{k} \vee \rho\right) \rightarrow T$ such that $\tilde{\lambda}\left([y]_{\zeta_{k}}\right)=\lambda_{1} g(y)$, for every $y \in B$. By the same argument given in the proof of Proposition 3.20, it is easily seen that $\tilde{\lambda}$ is a morphism in Preord*. We claim that the canonical image $\underline{\tilde{\lambda}}$ of $\widetilde{\lambda}$ in Preord ${ }^{*}$ is such that $\underline{\lambda}=\underline{\tilde{\lambda}} \pi$ (and it is clearly the unique one with this property, because $\underline{\pi}$ is an epimorphism by Proposition 3.15(3)). Since $\underline{\lambda}=\underline{\lambda_{1}} g$, there is a clopen subset $\Lambda$ of $(B, \rho)$ such that $\lambda=\lambda_{1} g$ on $\Lambda$ and both $\lambda$ and $\lambda_{1} g$ are trivial on $B \backslash \Lambda$. It easily follows that $\lambda=\tilde{\lambda} \pi$ on $\Lambda$ and that $\lambda, \tilde{\lambda} \pi$ are trivial on $B \backslash \Lambda$. This concludes the proof.

Proposition 4.2 An identity morphism $k:(A, \sigma) \rightarrow(A, \rho)$ is a prekernel of a morphism in

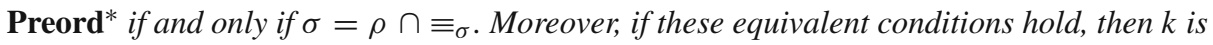
the prekernel of the canonical projection $\pi:(A, \rho) \rightarrow\left(A / \equiv_{\sigma}, \rho \vee \equiv_{\sigma}\right)$.

Proof If $k:(A, \sigma) \rightarrow(A, \rho)$ is a kernel of a morphism $f:(A, \rho) \rightarrow\left(A^{\prime}, \rho^{\prime}\right)$ in Preord, then $k$ is the morphism $k:\left(A, \rho \cap \sim_{f}\right) \rightarrow(A, \rho)$, where $\sim_{f}$ is the equivalence relation on $A$ defined, for every $x, y \in A$, by $x \sim_{f} y$ if $f(x)=f(y)$ and $k$ is the identity mapping. Thus $\sigma=\rho \cap \sim_{f}$. We must prove that $\sigma=\rho \cap \equiv_{\sigma}$. Now $\sigma=\rho \cap \sim_{f}$ implies that $\sigma \subseteq \rho$ and that $\sigma$ is contained in the equivalence relation $\sim_{f}$. Hence the equivalence relation $\equiv_{\sigma}$ generated by $\sigma$ is contained in $\sim_{f}$. Therefore, $\rho \cap \equiv_{\sigma} \subseteq \rho \cap \sim_{f}=\sigma$. Conversely, $\sigma \subseteq \rho$ because the identity $k:(A, \sigma) \rightarrow(A, \rho)$ is a morphism in Preord*, and $\sigma \subseteq \equiv_{\sigma}$ trivially. This concludes the proof of one of the implications of the first part of the statement. The rest follows trivially considering the canonical projection $\pi:(A, \rho) \rightarrow\left(A / \equiv_{\sigma}, \rho \vee \equiv_{\sigma}\right)$ and noticing that $\sim_{\pi}=\equiv_{\sigma}$.

The stable category $\underline{\text { Preord }}^{*}$ has an unexpected behaviour, because it has features typical of abelian categories and semiabelian categories (short exact sequences). A study of 
its properties (how far is it from being abelian?) is a promising starting point for further investigations. We are grateful to the referee for this and other remarks. We do not study the structure of the quotient category $\underline{\text { Preord }}^{*}$ in detail here, nor a possible more general setting where to define it, because it would lead us far from the subject dealt with in this paper.

Notice that similar questions and a similar construction of stable quotient category are given in [5], but the stable category presented in that article is not pointed.

Acknowledgements We are grateful to Marino Gran, Marco Grandis and Sandra Mantovani for some useful suggestions.

\section{Appendix: pretorsion theories}

We now briefly present a general setting for the results proved in the previous sections. We have called this section "Appendix" because the notion of pretorsion $(\mathcal{T}, \mathcal{F})$ in general categories we need here will be presented in further detail in the paper [4]. We have decided to call our pairs $(\mathcal{T}, \mathcal{F})$ "pretorsion" theories, because our concept properly generalizes a number of torsion theories existing in the mathematical literature. See, for instance, [6-8].

Our definition of pretorsion theory is based on the familiar idea (propagated by Marco Grandis, Sandra Mantovani and others) to fix a class $\mathcal{Z}$ of objects in a category $\mathcal{C}$ and declare a morphism to be $\mathcal{Z}$-trivial when it factors through an object in $\mathcal{Z}$. With these morphisms replacing the zero morphisms of a pointed category, one then arrives to our notions instantaneously. Letting $\mathcal{Z}$ be the class of discretely preordered sets, our example of pretorsion theory in the category Preord is easily checked and hardly surprising.

Torsion theory in general categories are studied in [6-8]. These papers are rather technical, and we will only use a tiny part of the results presented there. Hence, in this section, we give an elementary presentation of the results we need. There is a large body in the topologically oriented literature, which pursues the (torsion, torsion-free) paradigm in the form of general types of (connectedness, total disconnectedness). See, in particular, the papers [2,3] as well as the extensive literature cited there. The focus of these papers is on obtaining results in categories of topology that mirror, or even provide a common generalization of, the algebraic theory. Notice that, in our paper, the category of preordered sets is a topological category, a concept well studied in the literature [1] .

Fix an arbitrary category $\mathcal{C}$ and a class $\mathcal{Z}$ of objects of $\mathcal{C}$. For every pair $A, A^{\prime}$ of objects of $\mathcal{C}$, we will write $\operatorname{Triv}_{\mathcal{Z}}(A, B)$ for the set of all morphisms in $\mathcal{C}$ that factor through an object of $\mathcal{Z}$. We will call these morphisms $\mathcal{Z}$-trivial.

Let $f: A \rightarrow A^{\prime}$ be a morphism in $\mathcal{C}$. We say that a morphism $k: X \rightarrow A$ in $\mathcal{C}$ is a $\mathcal{Z}$-prekernel of $f$ if the following properties are satisfied:

1. $f k$ is a $\mathcal{Z}$-trivial morphism.

2. Whenever $\lambda: Y \rightarrow A$ is a morphism in $\mathcal{C}$ and $f \lambda$ is $\mathcal{Z}$-trivial, then there exists a unique morphism $\lambda^{\prime}: Y \rightarrow X$ in $\mathcal{C}$ such that $\lambda=k \lambda^{\prime}$.

Proposition 5.1 Let $f: A \rightarrow A^{\prime}$ be a morphism in $\mathcal{C}$ and let $\mu: X \rightarrow A$ be a $\mathcal{Z}$-prekernel for $f$. The following properties hold.

1. $\mu$ is a monomorphism.

2. If $\lambda: Y \rightarrow A$ is any other $\mathcal{Z}$-prekernel of $f$, then there exists a unique isomorphism $\lambda^{\prime}: Y \rightarrow X$ such that $\lambda=\mu \lambda^{\prime}$. 
Proof (1) Let $T$ be an object of $\mathcal{C}$ and $g, h: T \rightarrow X$ be morphisms in $\mathcal{C}$ such that $\mu g=\mu h$. Since $\mu$ is a $\mathcal{Z}$-prekernel of $f$, the morphism $f \mu$ is $\mathcal{Z}$-trivial, so that, a fortiori, the morphism $f \mu g$ is also $\mathcal{Z}$-trivial. By applying part (2) of the definition of $\mathcal{Z}$-prekernel to $\mu g$, it follows that there exists a unique morphism $u: T \rightarrow X$ such that $\mu u=\mu g=\mu h$. From the uniqueness of $u$, we get that $u=g=h$.

(2) By part (2) of the definition of $\mathcal{Z}$-prekernel, there is a unique morphism $\lambda^{\prime}: Y \rightarrow X$ such that $\lambda=\mu \lambda^{\prime}$ and a unique morphism $\mu^{\prime}: X \rightarrow Y$ such that $\mu=\lambda \mu^{\prime}$. It follows that $\lambda=\lambda \mu^{\prime} \lambda^{\prime}$ and $\mu=\mu \lambda^{\prime} \mu^{\prime}$. By part (1) of this proposition, we obtain that $\mu^{\prime} \lambda^{\prime}=1_{Y}, \lambda^{\prime} \mu^{\prime}=$ $1_{X}$. The conclusion is now clear.

Dually, a $\mathcal{Z}$-precokernel of $f$ is a morphism $p: A^{\prime} \rightarrow X$ such that:

1. $p f$ is a $\mathcal{Z}$-trivial morphism.

2. Whenever $\lambda: A^{\prime} \rightarrow Y$ is a morphism and $\lambda f$ is $\mathcal{Z}$-trivial, then there exists a unique morphism $\lambda_{1}: X \rightarrow Y$ with $\lambda=\lambda_{1} p$.

If $\mathcal{C}^{\text {op }}$ is the opposite category of $\mathcal{C}$, the $\mathcal{Z}$-precokernel of a morphism $f: A \rightarrow A^{\prime}$ in $\mathcal{C}$ is the $\mathcal{Z}$-prekernel of the morphism $f: A^{\prime} \rightarrow A$ in $\mathcal{C}^{\text {op }}$. Hence, from Proposition 5.1, we get:

Proposition 5.2 Let $f: A \rightarrow A^{\prime}$ be a morphism in a category $\mathcal{C}$ and let $p: A^{\prime} \rightarrow X$ be a $\mathcal{Z}$-precokernel of $f$. Then the following properties hold.

1. $p$ is an epimorphism.

2. If $q: A^{\prime} \rightarrow Y$ is another $\mathcal{Z}$-precokernel of $f$, then there exists a unique isomorphism $\varphi: X \rightarrow Y$ satisfying $q=\varphi p$.

Let $f: A \rightarrow B$ and $g: B \rightarrow C$ be morphisms in $\mathcal{C}$. We say that

$$
A \stackrel{f}{\longrightarrow} B \stackrel{g}{\longrightarrow} C
$$

is a short $\mathcal{Z}$-preexact sequence in $\mathcal{C}$ if $f$ is a $\mathcal{Z}$-prekernel of $g$ and $g$ is a $\mathcal{Z}$-precokernel of $f$.

Clearly, if $A \stackrel{f}{\longrightarrow} B \stackrel{g}{\longrightarrow} C$ is a short $\mathcal{Z}$-preexact sequence in $\mathcal{C}$, then $C \stackrel{g}{\longrightarrow} B \stackrel{f}{\longrightarrow} A$ is a short $\mathcal{Z}$-preexact sequence in $\mathcal{C}^{\text {op }}$.

It is now clear that if $\mathcal{C}:=$ Preord and $\mathcal{Z}$ is the class of all objects of type $(A,=)$ (where $A$ is any set and $=$ is the equality relation on $A$ ), then the $\mathcal{Z}$-prekernel and the $\mathcal{Z}$-precokernel of any morphism $f$ in Preord exist and coincide with the prekernel (resp., cokernel) of $f$. In particular, a short $\mathcal{Z}$-preexact sequence in Preord is a short preexact sequence, as in Definition 3.17 .

Definition 5.3 Let $\mathcal{C}$ be an arbitrary category. A pretorsion theory $(\mathcal{T}, \mathcal{F})$ for $\mathcal{C}$ consists of two classes $\mathcal{T}, \mathcal{F}$ of objects of $\mathcal{C}$, closed under isomorphism, satisfying the following two conditions. Set $\mathcal{Z}:=\mathcal{T} \cap \mathcal{F}$.

(1) For every object $B$ of $\mathcal{C}$ there is a short $\mathcal{Z}$-preexact sequence

$$
A \stackrel{f}{\longrightarrow} B \stackrel{g}{\longrightarrow} C
$$

with $A \in \mathcal{T}$ and $C \in \mathcal{F}$.

(2) $\operatorname{Hom}_{\mathcal{C}}(T, F)=\operatorname{Triv}_{\mathcal{Z}}(T, F)$ for every object $T \in \mathcal{T}, F \in \mathcal{F}$.

Lemma 5.4 Let $\mathcal{C}$ be a category, $\mathcal{Z}$ a class of objects of $\mathcal{C}$ and

$$
A \stackrel{f}{\longrightarrow} B \stackrel{g}{\longrightarrow} C
$$


a short $\mathcal{Z}$-preexact sequence. Then:

(a) $f$ is $\mathcal{Z}$-trivial if and only if $g$ is an isomorphism.

(b) $g$ is $\mathcal{Z}$-trivial if and only if $f$ is an isomorphism.

Proof (a) Suppose $f \mathcal{Z}$-trivial. By definition, the identity morphism $1_{B}: B \rightarrow B$ is clearly a $\mathcal{Z}$-precokernel of $f$. By the uniqueness up to isomorphism of $\mathcal{Z}$-precokernels (see Proposition 5.2), there is a unique isomorphism $h: C \rightarrow B$ such that $h g=1_{B}$. Hence $g$ is an isomorphism.

Conversely, assume that $g$ is an isomorphism. Since $g f$ is $\mathcal{Z}$-trivial, i.e. $g f$ factors though an object in $\mathcal{Z}$, the same holds for $f$, that is, $f$ is $\mathcal{Z}$-trivial.

(b) follows from (a) passing to the opposite category $\mathcal{C}^{\text {op }}$.

Proposition 5.5 Let $(\mathcal{T}, \mathcal{F})$ be a pretorsion theory in a category $\mathcal{C}$, let $\mathcal{Z}=\mathcal{T} \cap \mathcal{F}$, and let $X$ be any object in $\mathcal{C}$.

1. If $\operatorname{Hom}_{\mathcal{C}}(X, F)=\operatorname{Triv}_{\mathcal{Z}}(X, F)$ for every $F \in \mathcal{F}$, then $X \in \mathcal{T}$.

2. If $\operatorname{Hom}_{\mathcal{C}}(T, X)=\operatorname{Triv}_{\mathcal{Z}}(T, X)$ for every $T \in \mathcal{T}$, then $X \in \mathcal{F}$.

Proof By definition, there is a short $\mathcal{Z}$-preexact sequence $A \stackrel{f}{\longrightarrow} X \stackrel{g}{\longrightarrow} C$ with $A \in \mathcal{T}$ and $C \in \mathcal{F}$. If $\operatorname{Hom}_{\mathcal{C}}(X, F)=\operatorname{Triv}_{\mathcal{Z}}(X, F)$ for every $F \in \mathcal{F}$, then $g$ is $\mathcal{Z}$-trivial, so $f$ is an isomorphism by the previous lemma. As $\mathcal{T}$ is closed under isomorphism, we get that $X \in \mathcal{T}$. This proves (1). Similarly for (2).

Notice that if $(\mathcal{T}, \mathcal{F})$ is a pretorsion theory in $\mathcal{C}$, then $(\mathcal{F}, \mathcal{T})$ is a pretorsion theory in $\mathcal{C}^{\text {op }}$. Clearly, what we have proved in the previous sections shows that (Equiv,ParOrd) is a pretorsion theory in Preord.

Remark 5.6 It would be nice to try to characterize all pretorsion theories in the category Preord, but this seems to be hopeless. An example of pretorsion theory in Preord is given by the pair (ParOrd, Equiv). Notice that here we have inverted the order of the two subcategories with respect to what we have done until now in the article. Now the torsion objects are partially ordered sets, and the torsion-free objects are sets with an equivalence relation. In this case, the torsion-free quotient of an object $(A, \rho)$ of Preord is $\left(A, \equiv_{\rho}\right)$, where $\equiv_{\rho}$ is the equivalence relation described between the statements of Lemmas 2.8 and 2.9. (It is the transitive closure of the relation $\rho \rho^{\circ}$.) The torsion subobject of an object $(A, \rho)$ is the disjoint union of those clopen indecomposable subsets $[a]_{\equiv_{\rho}}$ of $(A, \rho)$ on which the preorder induced by $\rho$ is a partial order.

We consider this and other possible pretorsion theories on Preord much less interesting than that treated in the previous sections, which describes as any preordered set can be obtained from a partial order and an equivalence relation, in terms of a pretorsion theory.

Other easy natural examples of pretorsion theories in topological spaces, will be given in [4].

Remark 5.7 There is a notion of torsion theories for pointed categories given by Janelidze and Tholen in [8]. Their torsion theories are a special case of our notion of pretorsion theory. Namely, let $\mathcal{C}$ be a pointed category and let $\mathcal{Z}$ be the class of all zero objects on $\mathcal{C}$. Then, for any pair of fixed objects $A, B$ of $\mathcal{C}$, the family of all $\mathcal{Z}$-trivial morphisms consists exactly of all zero morphisms. It immediately follows that, if $f: A \rightarrow B, k: X \rightarrow A$ are morphisms in $\mathcal{C}$, then $k$ is a kernel of $f$ if and only if $k$ is a $\mathcal{Z}$-prekernel of $f$ (where $\mathcal{Z}$ is as before) and similarly for cokernels. Thus short exact sequences, as defined in [8], are exactly our short $\mathcal{Z}$-preexact sequences. In [8], a torsion theory in $\mathcal{C}$ is a pair $(\mathcal{T}, \mathcal{F})$ of full subcategories of $\mathcal{C}$ that are closed under isomorphism, satisfying the following axioms: 
1. $\operatorname{Hom}(T, F)=0$, for every $T \in \mathcal{T}, F \in \mathcal{F}$.

2. For every object $X$ in $\mathcal{C}$, there are objects $T(X) \in \mathcal{T}, F(X) \in \mathcal{F}$ and a short exact sequence $T(X) \rightarrow X \rightarrow F(X)$.

The fact that every torsion theory $(\mathcal{T}, \mathcal{F})$ is an example of pretorsion theory will follow if we prove that $\mathcal{T} \cap \mathcal{F}$ is exactly the class $\mathcal{Z}$ of all zero objects of $\mathcal{C}$. Clearly, every zero object is in $\mathcal{T} \cap \mathcal{F}$. Conversely, let $H$ be any object in $\mathcal{T} \cap \mathcal{F}$. By axiom (1), the identity $1_{H}: H \rightarrow H$ is the zero morphism, i.e. factors through a zero object. It immediately follows that $H$ is (isomorphic to) a zero object.

\section{References}

1. Adámek, J., Herrlich, H., Strecker, G.: "Abstract and Concrete Categories. The Joy of Cats", Pure and Applied Mathematics. Wiley, New York (1990)

2. Arhangel'ski, A.V., Wiegandt, R.: Connectednesses and disconnectednesses in topology. Gen. Topol. Appl. 5, 9-33 (1975)

3. Clementino, M.M., Tholen, W.: Separation versus connectedness. Topol. Appl. 75, 143-181 (1997)

4. Facchini, A., Finocchiaro, C., Gran, M.: Pretorsion Theories in General Categories, arXiv:1908.03546, 2019 (to appear)

5. Facchini, A., Heidari Zadeh, L.: An extension of properties of symmetric group to monoids and a pretorsion theory in a category of mappings. J. Algebra Appl. (2019). https://doi.org/10.1142/ S0219498819502347

6. Grandis, M., Janelidze, G.: From torsion theories to closure operators and factorization systems. Categ. Gen. Algebr. Struct. Appl. (2019) (to appear)

7. Grandis, M., Janelidze, G., Márki, L.: Non-pointed exactness, radicals, closure operators. J. Aust. Math. Soc. 94(3), 348-361 (2013)

8. Janelidze, G., Tholen, W.: Characterization of torsion theories in general categories. In: Davydov, A., Batanin, M., Johnson, M., Lack, S., Neeman, A. (eds.) Categories in Algebra, Geometry and Mathematical Physics, Providence, RI. Contemp. Math. Am. Math. Soc. 431, 249-256 (2007)

9. MacLane, S.: Categories for the Working Mathematician, 2nd edn. Springer, New York (1998)

10. Warfield Jr., R.B.: Serial rings and finitely presented modules. J. Algebra 37, 187-222 (1975)

Publisher's Note Springer Nature remains neutral with regard to jurisdictional claims in published maps and institutional affiliations. 